\title{
Dimethylcarbonate-assisted ring-opening of biobased valerolactones with methanol
}

\author{
Alessio Caretto, Marco Bortoluzzi, Maurizio Selva, Alvise Perosa* \\ Dipartimento di Scienze Molecolari e Nanosistemi, Università Ca’ Foscari Venezia, \\ Via Torino 155, 30172 Venezia Mestre, Italy
}

\section{Supporting information file (pages S1 to S12; 7 tables and 7 figures)}

Additional data for reactions carried out in batch conditions. .pag. S2

- Reactions carried out at low temperature (reflux of $\mathrm{CH}_{3} \mathrm{OH}$ or $\mathrm{DMC}$ ). ..pag. S2

- Reactions carried out at high temperature. ..pag. S2

Additional data for reactions carried out in continuous flow conditions pag. $\mathrm{S3}$

- Details about the apparatus and procedure. ..pag. S7

- Reaction data. ..pag. S7

Characterization. .pag. S10

\section{List of tables}

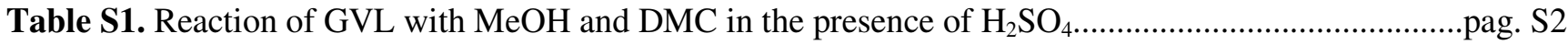

Table S2. Molar ratios of MeOH and DMC used respect to GVL .pag. $\mathrm{S} 2$

Table S3. Reaction between GVL and methanol, in the presence or absence of DMC, using scandium triflate as an acidic catalyst pag. S3

Table S4. Reactors assembled for the continuous flow study pag. S4

Table S5. Data and isolated yields for compound 2 (methyl 4-methoxypentanoate) for reactions between $\mathrm{GVL}$ and $\mathrm{CH}_{3} \mathrm{OH}$ over acidic alumina or $\mathrm{HY}$ zeolites for 15 hours of continuous sampling. .pag. S7

Table S6. Reaction between GVL and methanol over acidic alumina. Data collected at various flow rates and temperatures, in presence or absence of DMC pag. S8

Table S7. Reaction between GVL and methanol over HY zeolites. Data collected at various flow rates and temperatures, in presence or absence of DMC. pag. S9

\section{List of figures}

Figure S 1. Apparatus for performing continuous flow reactions ..pag. $\mathrm{S} 4$

Figure S 2. ${ }^{1} \mathrm{H}$ NMR spectrum of 2

Figure S 3. Mass spectrum of 2. .pag. S10

Figure S 4. Mass spectrum of $\mathbf{1}$ .pag. S11

Figure S 5. Mass spectrum of an isomer of $\mathbf{3}$ ..pag. S11

Figure S 6. Mass spectrum of an isomer of 3 . pag. S12

Figure S 7. Mass spectrum of an isomer of 3 . .pag. S12 


\section{Reactions carried out in batch}

Reactions at low temperature (reflux of $\mathrm{CH}_{3} \mathrm{OH}$ or DMC)

GVL (1.0 ml, $10.49 \mathrm{mmol})$ was loaded into a r.b. flask. Methanol (MeOH; $6.36 \mathrm{ml}, 157.31 \mathrm{mmol})$ and/or DMC (8.84 $\mathrm{ml}, 104.87 \mathrm{mmol})$ were added, with various molar ratios respect to GVL, as summarized in Table $\mathbf{S} \mathbf{1} \cdot \mathrm{H}_{2} \mathrm{SO}_{4}(79 \mathrm{mg}, 0.81 \mathrm{mmol})$ was added as an acid catalyst and the mixture was heated at reflux for 40 hours.

Table S 1. Reaction of GVL with $\mathrm{MeOH}$ and DMC in the presence of $\mathrm{H}_{2} \mathrm{SO}_{4}$.

\begin{tabular}{|c|c|c|c|c|c|c|c|}
\hline \multirow{2}{*}{ Entry $^{a}$} & \multirow{2}{*}{$\begin{array}{c}\mathrm{CH}_{3} \mathrm{OH} \\
(\mathrm{mol} / \mathrm{mol} \mathrm{GVL})\end{array}$} & \multirow{2}{*}{$\begin{array}{c}\text { DMC } \\
(\mathrm{mol} / \mathrm{mol} \mathrm{GVL})\end{array}$} & \multirow{2}{*}{$\begin{array}{c}\text { Temp. } \\
\left({ }^{\circ} \mathbf{C}\right)\end{array}$} & \multirow{2}{*}{$\begin{array}{c}\text { Conv. } \\
(\%)\end{array}$} & \multicolumn{3}{|c|}{ Selectivity $^{\mathrm{b}}(\%)$} \\
\hline & & & & & 1 & 2 & others ${ }^{c}$ \\
\hline 1 & 30 & - & 65 & 41 & 98 & 2 & - \\
\hline 2 & - & 30 & 90 & 1 & - & 100 & - \\
\hline 3 & 30 & 2 & 65 & 40 & 87 & 13 & - \\
\hline
\end{tabular}

${ }^{\mathrm{a}}$ Time $=40$ hours, $\mathrm{T}=90{ }^{\circ} \mathrm{C} . \mathrm{H}_{2} \mathrm{SO}_{4}=0.08 \mathrm{~mol} / \mathrm{mol} \mathrm{GVL} .{ }^{\mathrm{b}}$ Conversion and product percentages in the final mixture were determined by GC/MS analysis. ${ }^{\mathrm{c} O t h e r s ~ i n c l u d e ~ m a i n l y ~ m e t h y l p e n t e n o a t e s, ~ p l u s ~}$ traces of unidentified species.

\section{Reactions at high temperature}

GVL (1.0 ml, $10.49 \mathrm{mmol})$ was loaded into an autoclave. Methanol (MeOH; $6.36 \mathrm{ml}, 157.31 \mathrm{mmol})$ and/or DMC (8.84 $\mathrm{ml}, 104.87 \mathrm{mmol})$ were added, with various molar ratios respect to GVL, as summarized in Table $\mathbf{S} 2$. Initially reactions were performed with $\mathrm{H}_{2} \mathrm{SO}_{4}(79 \mathrm{mg}, 0.81 \mathrm{mmol})$ as an acid catalyst, while PTSA (140 mg, $0.81 \mathrm{mmol})$ was successively used.

Table S 2. Molar ratios of $\mathrm{MeOH}$ and DMC used respect to GVL.

\begin{tabular}{l|c|c|c|c|c|c}
\hline Entry & $\mathbf{1}$ & $\mathbf{2}$ & $\mathbf{3}$ & $\mathbf{4}$ & $\mathbf{5}$ & $\mathbf{6}$ \\
\hline $\mathrm{MeOH}(\mathrm{mol} / \mathrm{mol} \mathrm{GVL})$ & 30 & 30 & 15 & 10 & 2 & none \\
\hline $\mathrm{DMC}(\mathrm{mol} / \mathrm{mol} \mathrm{GVL})$ & none & 2 & 10 & 10 & 30 & 30 \\
\hline
\end{tabular}


The following Table S 3 summarizes results obtained for two experiments run using scandium triflate $\mathrm{ScTf}_{2}$. In the first experiment only $\mathrm{CH}_{3} \mathrm{OH}$ was used as a reactant, while in the second the ratio $\mathrm{CH}_{3} \mathrm{OH} / \mathrm{DMC} / \mathrm{GVL}$ was set to $15: 10: 1$. Both the reactions were performed at the following conditions: $\mathrm{T}=150{ }^{\circ} \mathrm{C}$, time $=24$ hours, $\mathrm{GVL}=1.0 \mathrm{~g}$.

Table S 3. Reaction between GVL and methanol, in the presence or absence of DMC, using scandium triflate as an acidic catalyst.

\begin{tabular}{|c|c|c|c|c|c|c|}
\hline \multirow{2}{*}{ Entry $^{a}$} & \multirow{2}{*}{$\begin{array}{c}\mathrm{CH}_{3} \mathrm{OH} \\
(\mathrm{mol} / \mathrm{mol} \mathrm{GVL})\end{array}$} & \multirow{2}{*}{$\begin{array}{c}\text { DMC } \\
(\mathrm{mol} / \mathrm{mol} \mathrm{GVL})\end{array}$} & \multirow{2}{*}{$\begin{array}{c}\text { Conv. } \\
(\%)\end{array}$} & \multicolumn{3}{|c|}{ Selectivity $(\%)^{b}$} \\
\hline & & & & 1 & 2 & others $^{c}$ \\
\hline 1 & 30 & None & 79 & 13 & 87 & - \\
\hline 2 & 15 & 10 & $>99$ & traces & 98 & 2 \\
\hline
\end{tabular}

\section{Reactions carried out in continuous flow conditions}

Details about the apparatus and procedure

Continuous flow reactions were performed in stainless steel tubular reactors (PFR) using the apparatus schematised in Figure S 1. The following equipment was employed: an HPLC pump Shimadzu LC-10AS; a GC oven HP 5890; a back pressure regulator (BPR) BP-2080 Plus. 


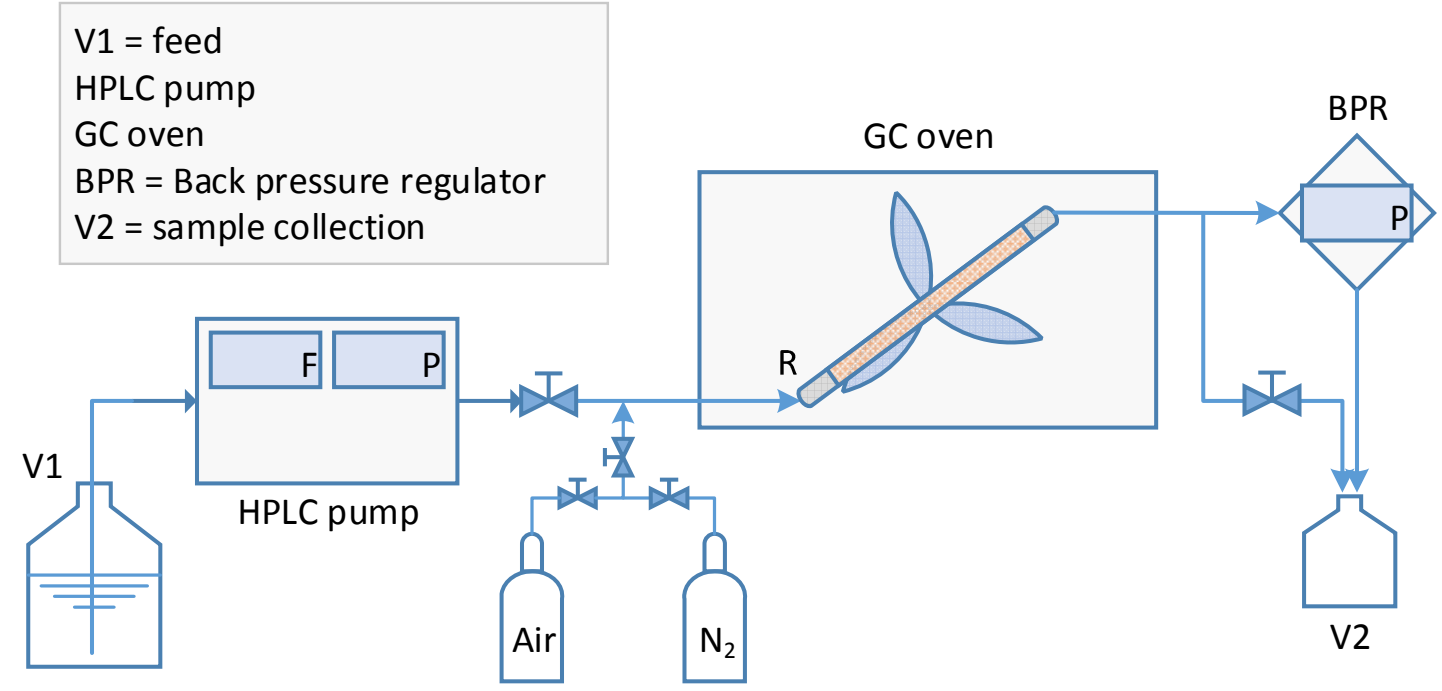

Figure S 1. Apparatus for performing continuous flow reactions.

All the connections between the apparatus components were realised with stainless steel tubes (internal diameter 1/16", unless otherwise stated).

Reactors were realised with Swagelok stainless steel tubes of $1 / 4$ " or $1 / 8$ " diameter. The various reactors assembled are listed in Table $\mathbf{S}$ 4. Both the reactor ends were fitted with Swagelok reducing unions (1/4-1/16" or 3/8-1/16"), together with two frits (Supelco; porosity = $2 \mu \mathrm{m}, \varnothing=1 / 4$ " or 3/8"), which allowed to connect the reactor to the pipeline.

Table S 4. Reactors assembled for the continuous flow study.

\begin{tabular}{c|c|c|c|c|c|c}
\hline Entry $^{\mathbf{a}}$ & \multicolumn{2}{|c|}{$\boldsymbol{\emptyset}_{\text {ext }}$} & \multicolumn{2}{c|}{$\boldsymbol{\emptyset}_{\text {int }}$} & $\begin{array}{c}\text { length } \\
\text { im }\end{array}$ & $\begin{array}{c}\text { volume } \\
\mathbf{c m}^{\mathbf{3}}\end{array}$ \\
\hline & inch & cm & inch & cm & cm & 1.40 \\
\hline 1 & $1 / 4$ & 0.635 & 0.15 & 0.385 & 12 & 3.65 \\
\hline 3 & $3 / 8$ & 0.953 & 0.25 & 0.623 & 12 & 20 \\
\hline
\end{tabular}

Preparation of the catalysts

Acidic alumina

Acidic alumina was dried before use as following described. The solid powder was loaded into the chosen reactor for the maximum capability of the latter. The reactor was then assembled in the 
apparatus described above, a flow of nitrogen was applied $\left(15 \mathrm{ml} \mathrm{min}^{-1}\right)$ and the temperature was raised to $250{ }^{\circ} \mathrm{C}$ (8 hours). The so prepared alumina was either used fresh or stored at $70{ }^{\circ} \mathrm{C}$ under vacuum.

\section{HY zeolites}

HY zeolites were prepared by calcination of $\mathrm{NH}_{4} \mathrm{Y}$ zeolites. $8 \mathrm{~g}$ of the latter were placed in a quartz tube, placed in a vertical oven. A flow of dry air $\left(25 \mathrm{ml} \mathrm{min}^{-1}\right)$ was applied from the top of the tube, which was then heated up to $500{ }^{\circ} \mathrm{C}$ for 5 hours. The so prepared HY zeolites were either used fresh or stored at $70{ }^{\circ} \mathrm{C}$ under vacuum.

\section{Start-up procedure}

i. The HPLC pump was set to the desired flow rate $\left(\mathrm{ml} \mathrm{min}^{-1}\right)$, while the BPR was set on atmospheric pressure.

ii. When collection started at the outlet, the desired pressure was set on the BPR.

iii. Once the system reached the equilibrium (constant pressure both at the pump and at the $\mathrm{BPR})$, the temperature in the oven was increased by programming a temperature $\operatorname{ramp}\left(5^{\circ} \mathrm{C}\right.$ $\min ^{-1}$ up to the desired temperature).

iv. Once the desired temperature was reached, and the system reached the new equilibrium (constant pressures and temperature), the apparatus was kept operating for a certain time (equilibration time), the latter calculated by considering an estimated contact time into the reactor and an estimated total time for the mixture to flow across all the pipeline.

v. After the equilibration time, the sampling of the mixture at the outlet was started. Single samples were collected for 30 minutes and analysed at the GC-MS. At least 3 samples (total sampling time of 90 minutes) were collected for every set of conditions. When little discrepancy in the product distribution between the samples was observed, a fourth and a fifth fractions were collected. All the data reported in chapter 4 are an average of the samples collected for that single set of conditions. 
vi. When a single parameter was changed, point iv (equilibration time) and v (sampling) were repeated as described.

\section{Shut down procedure}

Steps followed to stop the apparatus at the end of a reaction.

i. The temperature was decreased to r.t. by applying a temperature ramp of $-3{ }^{\circ} \mathrm{C} \min ^{-1}$.

ii. Once the $\mathrm{GC}$ oven reached a temperature of $35-40{ }^{\circ} \mathrm{C}$, the $\mathrm{BPR}$ was set to atmospheric pressure.

iii. The feed mixture was replaced by methanol, fed at a flow rate of $2 \mathrm{ml} \mathrm{min} \mathrm{m}^{-1}$ and the whole line was purged for 1 hour. After this time the outlet mixture was sampled and analysed at the GC-MS. When traces of reactant/products were detected, purging was continued for intervals of 30 minutes and systematically sampled, until when the outlet mixture became clean.

iv. The pump was switched off and the valves were closed.

v. three different sets of conditions $\left(180{ }^{\circ} \mathrm{C}, 0.02 \mathrm{ml} / \mathrm{min} ; 180{ }^{\circ} \mathrm{C}, 0.05 \mathrm{ml} / \mathrm{min} ; 250{ }^{\circ} \mathrm{C}, 0.05\right.$ $\mathrm{ml} / \mathrm{min}$ ) 
Reaction data

Table S 5. Data and isolated yields for compound 2 (methyl 4-methoxypentanoate) for reactions between $\mathrm{GVL}$ and $\mathrm{CH}_{3} \mathrm{OH}$ over acidic alumina or $\mathrm{HY}$ zeolites for 15 hours of continuous sampling.

\begin{tabular}{|c|c|c|c|c|c|c|c|c|c|}
\hline \multirow[t]{2}{*}{ Entry $^{a}$} & \multirow[t]{2}{*}{ Catalyst } & \multirow{2}{*}{$\begin{array}{c}\mathrm{DMC} / \\
\mathrm{CH}_{3} \mathrm{OH} \\
(\mathrm{vol} / \mathrm{vol})\end{array}$} & \multirow{2}{*}{$\begin{array}{c}\text { Flow } \\
\text { rate } \\
(\mathrm{ml} / \mathrm{min})\end{array}$} & \multirow{2}{*}{$\begin{array}{l}\text { Temp. } \\
\left({ }^{\circ} \mathrm{C}\right)\end{array}$} & \multirow{2}{*}{$\begin{array}{c}\text { Conv. } \\
(\%)\end{array}$} & \multicolumn{3}{|c|}{$\begin{array}{c}\text { Yield }^{\mathrm{b}} \\
(\text { GC-MS \%) }\end{array}$} & \multirow{2}{*}{$\begin{array}{c}\text { Isolated } \\
\text { yield of } \\
2(\%)\end{array}$} \\
\hline & & & & & & 1 & 2 & 3 & \\
\hline 1 & $\begin{array}{c}\text { HY } \\
\text { zeolites }\end{array}$ & 0 & 0.02 & 180 & 77 & 2 & 71 & 4 & 60 \\
\hline 2 & $\begin{array}{c}\text { HY } \\
\text { zeolites }\end{array}$ & 0 & 0.05 & 180 & 66 & 6 & 60 & $<1$ & 55 \\
\hline 3 & $\begin{array}{l}\text { Acidic } \\
\text { alumina }\end{array}$ & 0.33 & 0.05 & 250 & 55 & 2 & 52 & 1 & 46 \\
\hline
\end{tabular}

${ }^{\mathrm{a}}$ Pressure: 60 bar. ${ }^{\mathrm{b}}$ Conversion and product percentages in the final mixture were determined by GC/MS analysis. 
Table S 6. Reaction between GVL and methanol over acidic alumina. Data collected at various flow rates and temperatures, in presence or absence of DMC.

\begin{tabular}{|c|c|c|c|c|c|c|c|}
\hline \multirow[t]{2}{*}{ Entry } & \multirow{2}{*}{$\begin{array}{c}\text { mix. } \\
\text { composition }\end{array}$} & \multirow{2}{*}{$\begin{array}{l}\text { FLOW } \\
(\mathrm{ml} / \mathrm{min})\end{array}$} & \multirow{2}{*}{$\begin{array}{c}\mathbf{T} \\
\left({ }^{\circ} \mathbf{C}\right)\end{array}$} & \multirow{2}{*}{$\begin{array}{c}\text { Conv. }^{b} \\
(\%)\end{array}$} & \multicolumn{3}{|c|}{ Prod. $^{\mathrm{b}}(\%)$} \\
\hline & & & & & 4.1 & 4.2 & 4.3 \\
\hline 1 & \multirow{8}{*}{$\begin{array}{c}\text { MeOH } \\
\text { only }\end{array}$} & \multirow[t]{4}{*}{0.25} & 180 & 7 & 7 & $<1$ & 0 \\
\hline 2 & & & 200 & 7 & 7 & $<1$ & 0 \\
\hline 3 & & & 220 & 8 & 5 & 3 & 0 \\
\hline 4 & & & 250 & 14 & 9 & 5 & 0 \\
\hline 5 & & \multirow[t]{4}{*}{0.1} & 180 & 15 & 14 & $<1$ & 0 \\
\hline 6 & & & 200 & 15 & 14 & 1 & 0 \\
\hline 7 & & & 220 & 15 & 12 & 3 & 0 \\
\hline 8 & & & 250 & 22 & 9 & 13 & 0 \\
\hline
\end{tabular}

\begin{tabular}{|c|c|c|c|c|c|c|c|}
\hline & \multirow{2}{*}{$\begin{array}{c}\text { mix. } \\
\text { composition }\end{array}$} & \multirow{2}{*}{$\begin{array}{l}\text { FLOW } \\
(\mathrm{ml} / \mathrm{min})\end{array}$} & \multirow{2}{*}{$\begin{array}{c}\mathbf{T} \\
\left({ }^{\circ} \mathbf{C}\right)\end{array}$} & \multirow{2}{*}{$\begin{array}{c}\text { Conv. }^{b} \\
(\%)\end{array}$} & \multicolumn{3}{|c|}{ Prod. $^{b}(\%)$} \\
\hline & & & & & 4.1 & 4.2 & 4.3 \\
\hline 9 & \multirow{8}{*}{$\begin{array}{c}M e O H: D M C \\
=3: 1\end{array}$} & \multirow[t]{4}{*}{0.25} & 180 & 8 & 7 & 1 & 0 \\
\hline 10 & & & 200 & 8 & 7 & 1 & 0 \\
\hline 11 & & & 220 & 10 & 6 & 4 & 0 \\
\hline 12 & & & 250 & 29 & 5 & 23 & 1 \\
\hline 13 & & \multirow[t]{4}{*}{0.1} & 180 & 10 & 10 & $<1$ & 0 \\
\hline 14 & & & 200 & 13 & 11 & 2 & 0 \\
\hline 15 & & & 220 & 16 & 8 & 8 & 0 \\
\hline 16 & & & 250 & 41 & 5 & 35 & 1 \\
\hline
\end{tabular}

\begin{tabular}{|c|c|c|c|c|c|c|c|}
\hline & \multirow{2}{*}{$\begin{array}{c}\text { mix. } \\
\text { composition }\end{array}$} & \multirow{2}{*}{$\begin{array}{l}\text { FLOW } \\
(\mathrm{ml} / \mathrm{min})\end{array}$} & \multirow{2}{*}{$\begin{array}{c}\mathbf{T} \\
\left({ }^{\circ} \mathbf{C}\right)\end{array}$} & \multirow{2}{*}{$\begin{array}{c}\text { Conv. }^{b} \\
(\%)\end{array}$} & \multicolumn{3}{|c|}{ Prod. ${ }^{b}(\%)$} \\
\hline & & & & & 4.1 & 4.2 & 4.3 \\
\hline 17 & \multirow{9}{*}{$\begin{array}{c}M e O H: D M C \\
\quad=1: 1\end{array}$} & \multirow[t]{4}{*}{0.25} & 180 & 7 & 6 & 1 & 0 \\
\hline 18 & & & 200 & 7 & 5 & 2 & 0 \\
\hline 19 & & & 220 & 14 & 5 & 9 & 0 \\
\hline 20 & & & 250 & 43 & 2 & 40 & 1 \\
\hline 21 & & \multirow[t]{4}{*}{0.1} & 180 & 8 & 11 & $<1$ & 0 \\
\hline 22 & & & 200 & 11 & 9 & 2 & 0 \\
\hline 23 & & & - & - & - & - & - \\
\hline 24 & & & 250 & 52 & 2 & 48 & 2 \\
\hline 25 & & 0.05 & 250 & 55 & 2 & 52 & 1 \\
\hline
\end{tabular}

${ }^{a}$ Reactions were carried out at a pressure of 60 bar. ${ }^{b}$ Conversions and product percentages in the final mixture were determined by GC/MS analysis. 
Table S 7. Reaction between GVL and methanol over HY zeolites. Data collected at various flow rates and temperatures, in presence or absence of DMC.

\begin{tabular}{|c|c|c|c|c|c|c|c|}
\hline \multirow[t]{2}{*}{ Entry } & \multirow{2}{*}{$\begin{array}{c}\text { mix. } \\
\text { composition }\end{array}$} & \multirow{2}{*}{$\begin{array}{l}\text { FLOW } \\
(\mathrm{ml} / \mathrm{min})\end{array}$} & \multirow{2}{*}{$\begin{array}{c}\mathbf{T} \\
\left({ }^{\circ} \mathbf{C}\right)\end{array}$} & \multirow{2}{*}{$\begin{array}{c}\text { Conv. }^{b} \\
(\%)\end{array}$} & \multicolumn{3}{|c|}{ Prod. $^{b}$} \\
\hline & & & & & 4.1 & 4.2 & 4.3 \\
\hline 1 & \multirow{9}{*}{$\begin{array}{c}\text { MeOH } \\
\text { only }\end{array}$} & \multirow[t]{4}{*}{0.25} & 180 & 45 & 6 & 37 & 2 \\
\hline 2 & & & 200 & 57 & 3 & 48 & 6 \\
\hline 3 & & & 220 & 57 & 2 & 43 & 12 \\
\hline 4 & & & 250 & 63 & 2 & 18 & 43 \\
\hline 5 & & \multirow[t]{4}{*}{0.1} & 180 & 51 & 6 & 41 & 4 \\
\hline 6 & & & 200 & 59 & 4 & 46 & 9 \\
\hline 7 & & & 220 & 50 & 4 & 28 & 18 \\
\hline 8 & & & 250 & 53 & 2 & 8 & 43 \\
\hline 9 & & 0.05 & 180 & 66 & 6 & 60 & $>1$ \\
\hline
\end{tabular}

\begin{tabular}{|c|c|c|c|c|c|c|c|}
\hline \multirow[t]{2}{*}{ Entry } & \multirow{2}{*}{$\begin{array}{c}\text { mix. } \\
\text { composition }\end{array}$} & \multirow{2}{*}{$\begin{array}{c}\text { FLOW } \\
(\mathrm{ml} / \mathrm{min})\end{array}$} & \multirow{2}{*}{$\begin{array}{l}\mathbf{T} \\
\left({ }^{\circ} \mathbf{C}\right)\end{array}$} & \multirow{2}{*}{$\begin{array}{c}\text { Conv. }^{\mathrm{b}} \\
(\%)\end{array}$} & \multicolumn{3}{|c|}{ Prod. $^{b}$} \\
\hline & & & & & 4.1 & 4.2 & 4.3 \\
\hline 10 & MeOH:DMC & 0.25 & 180 & 42 & 5 & 37 & $<1$ \\
\hline 11 & $=3: 1$ & & 200 & 60 & 4 & 53 & 3 \\
\hline 12 & & & 220 & 69 & 3 & 56 & 10 \\
\hline 13 & & & 250 & 74 & 1 & 21 & 51 \\
\hline 14 & & 0.10 & 180 & 56 & 4 & 52 & 2 \\
\hline 15 & & & 200 & 74 & 3 & 66 & 5 \\
\hline 16 & & & 220 & 71 & 2 & 53 & 16 \\
\hline 17 & & & 250 & 65 & 1 & 7 & 53 \\
\hline 18 & & 0.02 & 180 & 76 & 3 & 70 & 3 \\
\hline
\end{tabular}

\begin{tabular}{|c|c|c|c|c|c|c|c|}
\hline \multirow[t]{2}{*}{ Entry } & \multirow{2}{*}{$\begin{array}{c}\text { mix. } \\
\text { composition }\end{array}$} & \multirow{2}{*}{$\begin{array}{l}\text { FLOW } \\
(\mathrm{ml} / \mathrm{min})\end{array}$} & \multirow{2}{*}{$\begin{array}{c}\mathbf{T} \\
\left({ }^{\circ} \mathbf{C}\right)\end{array}$} & \multirow{2}{*}{$\begin{array}{c}\text { Conv. }^{\text {b }} \\
(\%)\end{array}$} & \multicolumn{3}{|c|}{ Prod. $^{\mathrm{b}}$} \\
\hline & & & & & 4.1 & 4.2 & 4.3 \\
\hline 19 & \multirow{10}{*}{$\begin{array}{c}\mathrm{MeOH}: \mathrm{DMC} \\
\quad=1: 1\end{array}$} & \multirow[t]{4}{*}{0.25} & 180 & 45 & 4 & 40 & 1 \\
\hline 20 & & & 200 & 63 & 1 & 57 & 5 \\
\hline 21 & & & 220 & 71 & 1 & 53 & 17 \\
\hline 22 & & & 250 & 76 & $<1$ & 12 & 62 \\
\hline 23 & & \multirow[t]{4}{*}{0.1} & 180 & 60 & 4 & 55 & 1 \\
\hline 24 & & & 200 & 76 & 2 & 66 & 8 \\
\hline 25 & & & 220 & 72 & 2 & 56 & 14 \\
\hline 26 & & & 250 & 74 & 1 & 7 & 65 \\
\hline 27 & & 0.05 & 180 & 66 & 2 & 60 & 3 \\
\hline 28 & & 0.02 & 180 & 81 & 2 & 73 & 6 \\
\hline
\end{tabular}

${ }^{a}$ Reactions were carried out at a pressure of 60 bar. ${ }^{b}$ Conversions and product percentages in the final mixture were determined by GC/MS analysis. 


\section{Characterization}

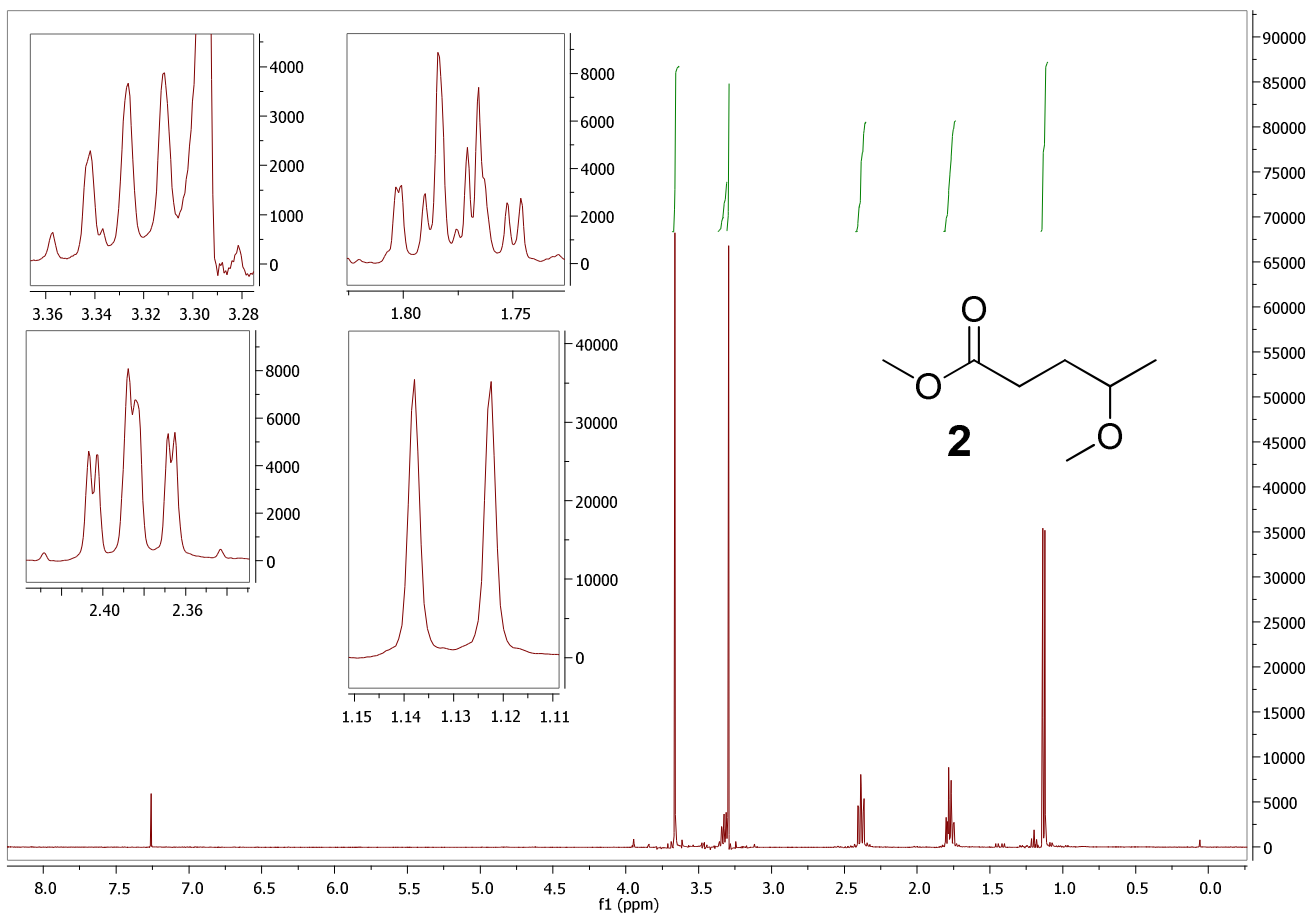

Figure S 2. ${ }^{1} \mathrm{H}$ NMR spectrum of 2 .

${ }^{1} \mathrm{H}$ NMR (400 MHz, CDCl3) $\delta 3.66(\mathrm{~s}, 3 \mathrm{H}), 3.32(\mathrm{~m}, 1 \mathrm{H}), 3.29(\mathrm{~s}, 3 \mathrm{H}), 2.38(\mathrm{~m}, 2 \mathrm{H}), 1.77(\mathrm{~m}, 2 \mathrm{H})$, $1.13(\mathrm{~d}, 3 \mathrm{H}, \mathrm{J}=6.2)$.

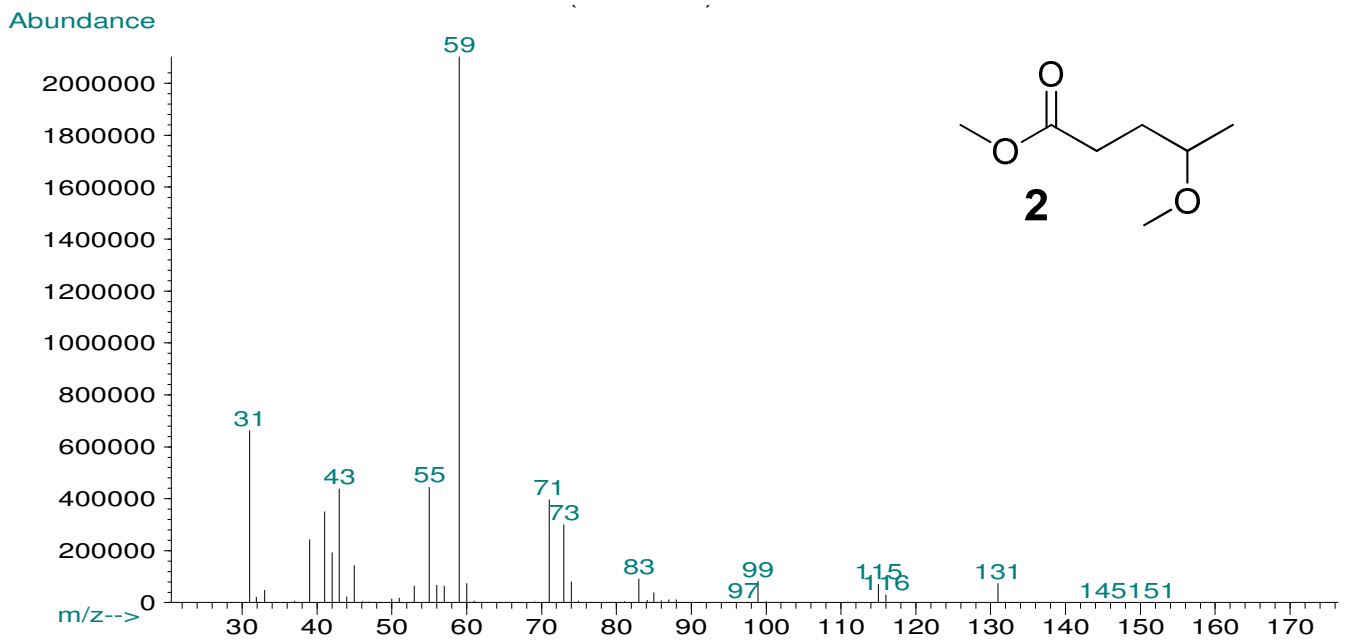

Figure S 3. Mass spectrum of 2.

GC-MS (relative intensity, $70 \mathrm{eV}) \mathrm{m} / \mathrm{z}: 146\left(\mathrm{M}^{+},<1 \%\right), 131\left(\mathrm{M}^{+}-\mathrm{CH}_{3},\right), 115\left(\mathrm{M}^{+}-\mathrm{OCH}_{3}, 4\right), 99$ $\left(\left[\mathrm{C}_{5} \mathrm{H}_{7} \mathrm{O}_{2}\right]^{+}, 4\right), 83$ (4), 73 (14), 71 (18), 59 ([COOCH$\left.]^{+}, 100\right), 55\left(\left[\mathrm{C}_{3} \mathrm{H}_{3} \mathrm{O}\right]^{+}, 20\right), 43$ (19), 41 (15), 39 (10), $31(31)$. 


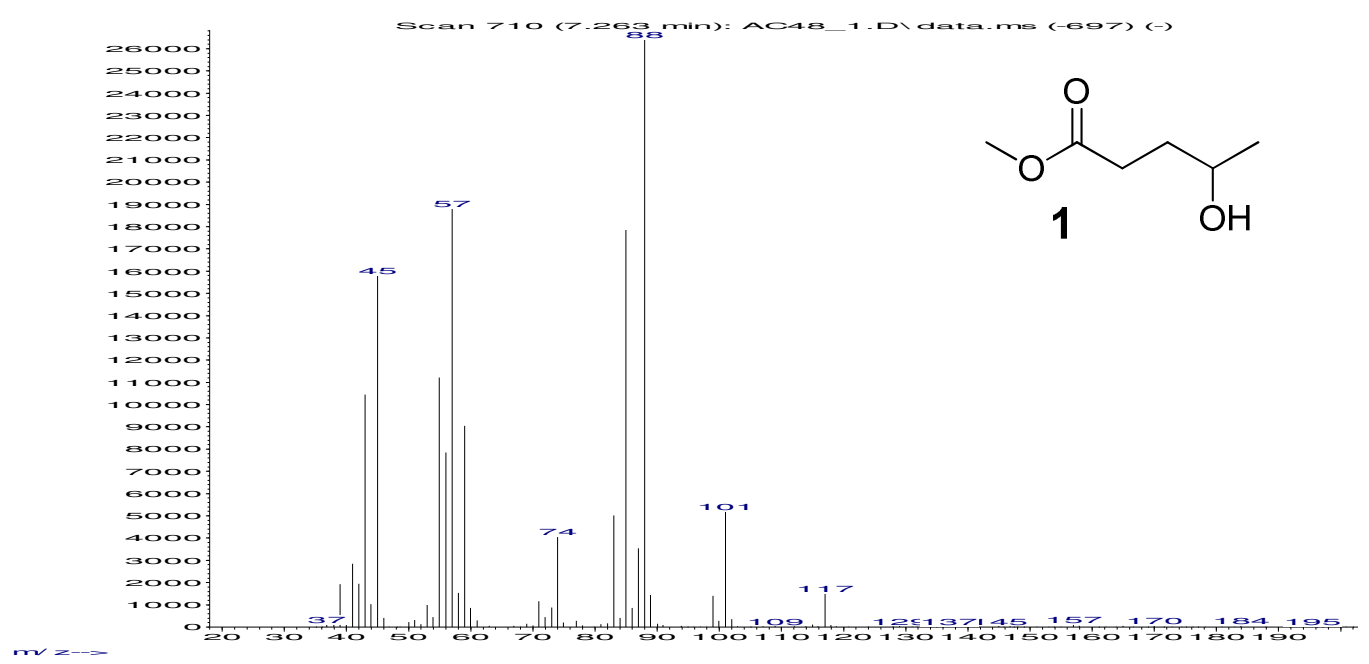

Figure S 4. Mass spectrum of 1 .

GC-MS (relative intensity, $70 \mathrm{eV}$ ) m/z: $132\left([\mathrm{M}]^{+}<1 \%\right), 117$ (6), 101 (19), 99 (5), 88 (100), 87 (13), 85 (66), 83 (19), 74 (15), 59 (34), 58 (6), 57 (70), 56 (29), 55 (42), 45 (59), 43 (39), 42 (7), 41 (11).

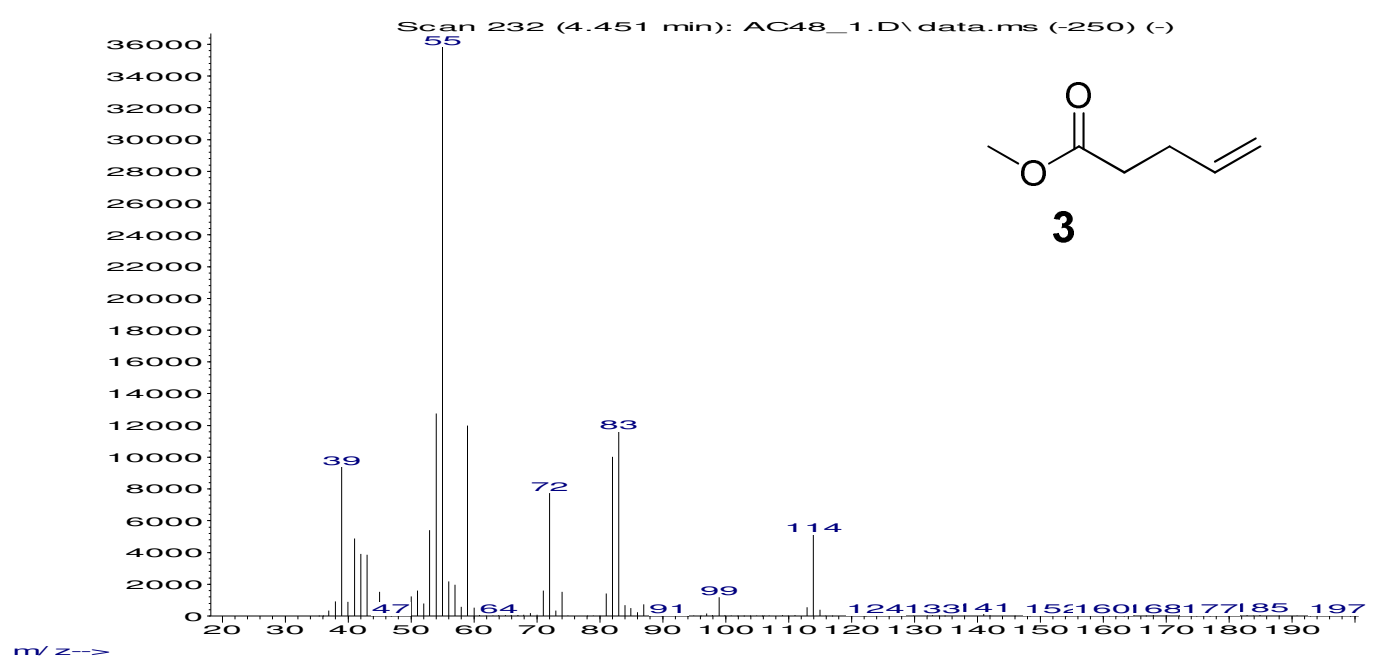

Figure S 5. Mass spectrum of an isomer of $\mathbf{3}$.

GC-MS (relative intensity, $70 \mathrm{eV}$ ) m/z: 114 ([M] $\left.{ }^{+}, 13 \%\right), 99$ (3), 83 (30), 82 (26), 72 (20), 59 (32), 56 (6), 55 (100), 54 (37), 53 (14), 43 (9), 42 (10), 41 (13), 39 (26). 


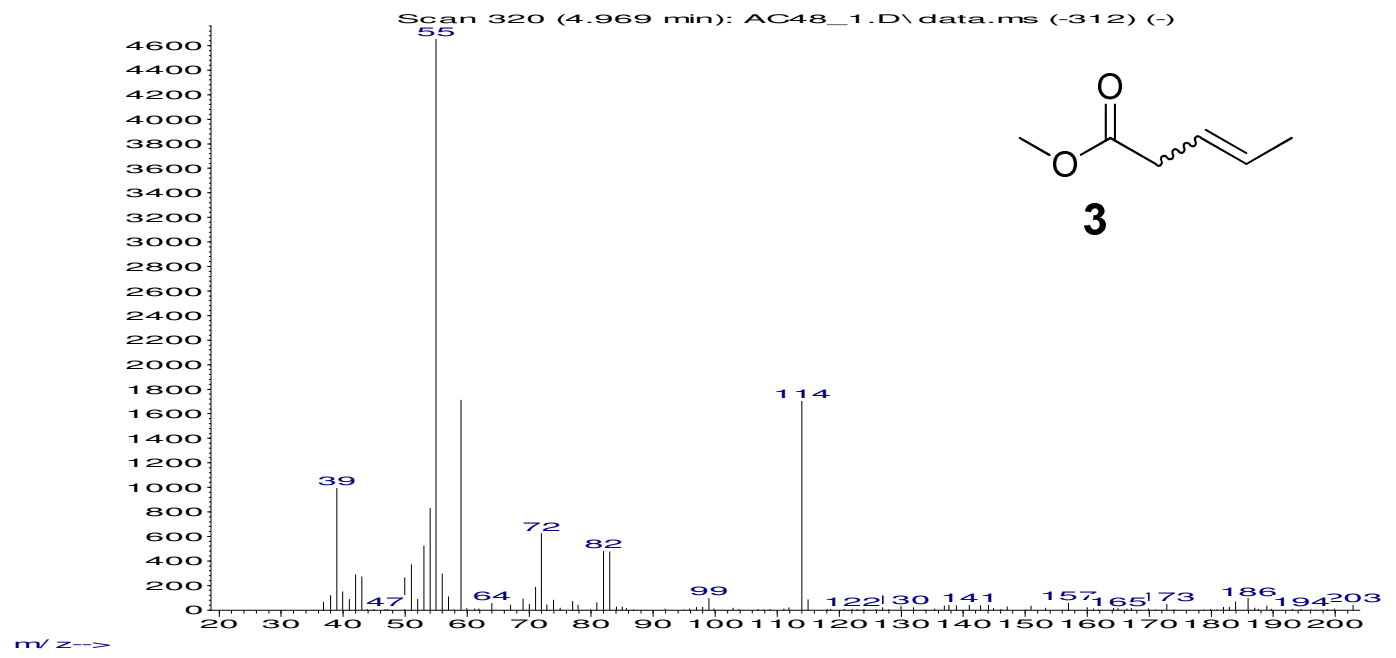

Figure S 6. Mass spectrum of an isomer of 3.

GC-MS (relative intensity, $70 \mathrm{eV}$ ) m/z: $114\left(\left[\mathrm{M}^{+}, 34 \%\right), 99\right.$ (3), 83 (8), 82 (14), 72 (16), 59 (33), 55 (100), 54 (17), 53 (11), 51 (7), 43 (5), 42 (5), 39 (23).

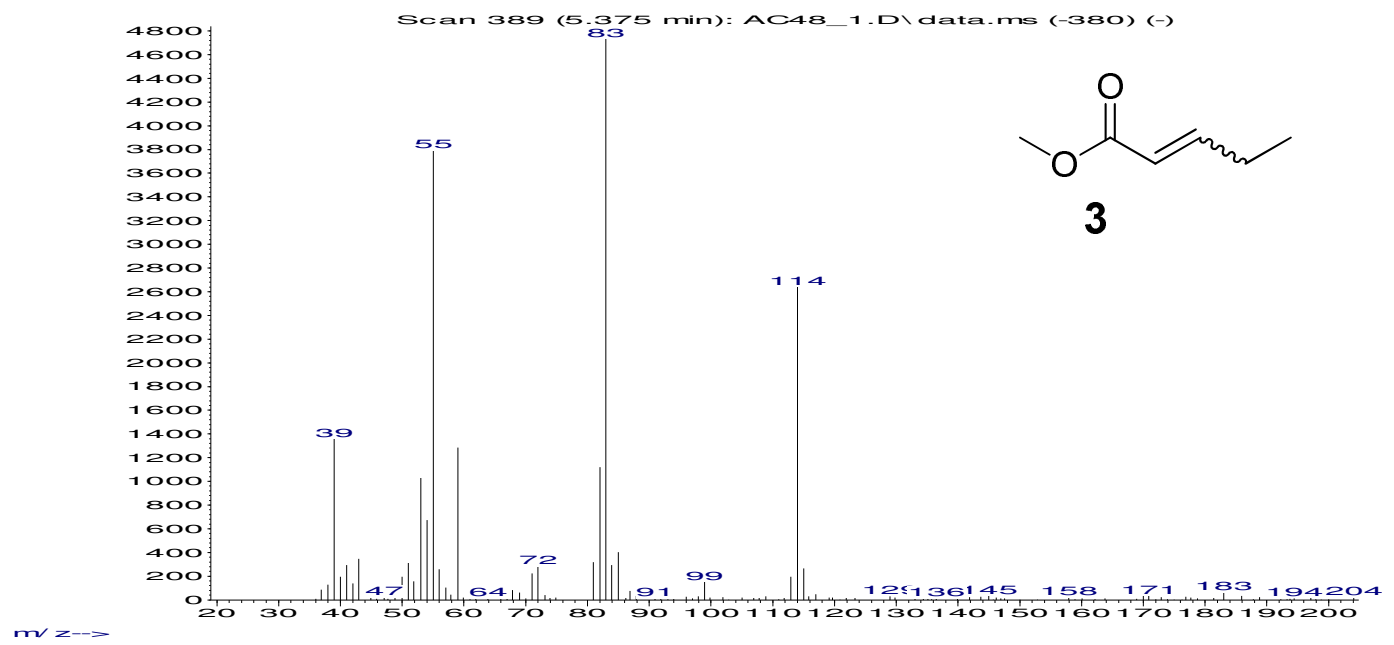

Figure S 7. Mass spectrum of an isomer of $\mathbf{3}$.

GC-MS (relative intensity, $70 \mathrm{eV}) \mathrm{m} / \mathrm{z}: 114$ ([M] $\left.]^{+}, 59 \%\right), 99$ (5), 85 (6), 84 (6), 83 (100), 82 (21), 81 (6), 72 (5), 71 (6), 59 (26), 56 (5), 55 (81), 54 (14), 53 (20), 51 (8), 43 (10), 39 (25). 